\title{
Drug Accountability Result Standard Unit
}

National Cancer Institute

\section{Source}

National Cancer Institute. Drug Accountability Result Standard Unit. NCI Thesaurus. Code C87875.

The standard unit of measure for drug accountability results. 